\title{
Assessment and Reproducibility of Non-Eosinophilic Asthma Using Induced Sputum
}

\author{
Jodie L. Simpson ${ }^{\mathrm{a}-\mathrm{c}}$ Patrick McElduff ${ }^{c}$ Peter G. Gibson ${ }^{\mathrm{a}-\mathrm{c}}$ \\ ${ }^{a}$ Centre for Asthma and Respiratory Disease and ${ }^{b}$ School of Medicine and Public Health, The University of \\ Newcastle, Callaghan, N.S.W., and ' Department of Respiratory and Sleep Medicine, Hunter Medical Research \\ Institute, John Hunter Hospital, New Lambton, N.S.W., Australia
}

\section{Key Words}

Asthma $\cdot$ Eosinophils $\cdot$ Non-eosinophilic asthma $\cdot$ Induced sputum $\cdot$ Reproducibility

\begin{abstract}
Background: The assessment of eosinophilic airway inflammation using induced sputum identifies a corticosteroid-responsive subtype that can be used to guide anti-inflammatory therapy. The stability of airway inflammation in asthma over time is not known, yet this information is crucial to inflammation-based patient management. Objective: The aim of this study was to determine the reproducibility of non-eosinophilic asthma. Methods: Participants with stable asthma $(n=26)$ underwent a sputum induction each month for 5 months. Sputum was dispersed and differential cell counts were performed. The reproducibility of inflammatory subtype with different eosinophil cut points (starting at $1 \%$ eosinophils) was examined and the minimum number of visits required to determine inflammatory subtype was calculated. Results: One hundred and twentytwo sputum samples were obtained (success $94 \%$ ). All cut points greater than $2 \%$ eosinophils were reproducible and a $3 \%$ cut point resulted in the highest agreement with a $\mathrm{k}$ statistic of 0.538 . Specificity and sensitivity were high for determining inflammatory subtype after 1 or 3 sputum samples. Conclusions: A cut point of $3 \%$ eosinophils should be used to distinguish eosinophilic from non-eosinophilic
\end{abstract}

airway inflammation in asthma. This allows a single sputum sample to be used to reliably determine the presence of eosinophilia.

Copyright $\odot 2009$ S. Karger AG, Basel

\section{Introduction}

Airway inflammation in asthma is heterogeneous, and non-eosinophilic forms of asthma occur frequently in both adults and children [1-3]. Non-eosinophilic asthma has been identified in the airways using both induced sputum [4] and endobronchial biopsies [5]. The distinction between eosinophilic and non-eosinophilic asthma is made using a specific proportion of eosinophils (the cut point), generally set as the upper limit of normal. A number of cut points have been reported which are calculated from the upper limits from a healthy population. We have recently found that a cut point of $1 \%$ [4] can distinguish between asthma subtypes whereas cut points between 2 and 3\% have been shown to be useful in decisions about corticosteroid treatment $[6,7]$.

Inflammation-based asthma management is an emerging treatment paradigm that promises improved asthma control with the same or lower dose of corticosteroid therapy. Induced sputum is a simple, validated, noninvasive technique to assess airway inflammation. The 
utility of sputum eosinophil counts has been recently demonstrated in 2 randomised controlled trials that compared traditional management strategies based upon symptoms and lung function with a management strategy based upon normalisation of sputum eosinophil counts. The identification of eosinophilic and non-eosinophilic asthma and subsequent management-based sputum eosinophil counts resulted in both fewer and less severe exacerbations $[6,7]$.

The reproducibility of inflammatory subtypes over time is unknown and the identification of subtypes is generally made from a single sputum sample collected in a cross-sectional setting. The aim of this study was to determine the reproducibility of non-eosinophilic asthma and examine the effect of the eosinophil cut point on reproducibility. In addition, we sought to estimate the minimum number of samples required to determine inflammatory subtype.

\section{Methods}

\section{Participants}

Participants in this study formed the placebo arm of a randomised controlled trial which is reported elsewhere [8]. Nonsmoking adults ( $<5$ pack-years) with symptomatic refractory asthma ( $\mathrm{n}=26)$, defined according to GINA guidelines [9] with demonstrated airway hyperresponsiveness, were studied. Participants were recruited from the Ambulatory Care service of the Department of Respiratory and Sleep Medicine at the John Hunter Hospital. During the study, maintenance asthma medications remained stable and were used by participants as prescribed by their physician. No participant required an alteration in asthma therapy, course of oral corticosteroids or hospital admission throughout the study.

Design

The Hunter Area Health Service and The University of Newcastle Human Research Ethics Committees approved this longitudinal study. Participants were invited to attend the research clinic once each month for a total of 5 months. At each visit participants underwent a clinical assessment, spirometry and combined hypertonic saline challenge and sputum induction [10]. Induced sputum was processed for inflammatory cell counts.

\section{Methods}

Sputum Induction

Spirometry (KoKo PD Instrumentation, Louisville, Colo., USA) and combined bronchial provocation testing and sputum induction with hypertonic saline (4.5\%) were performed as previously described [10]

\section{Sputum Processing and Cell Counts}

The sputum was selected from saliva and dispersed using dithiothreitol as described previously [10]. The suspension was filtered, and a total cell count of leukocytes and viability was per- formed. Cytospins were stained (May-Grunwald Geimsa) and a differential cell count was obtained from 400 non-squamous cells. An adequate sputum sample was one that returned a cell count from more than 400 non-squamous cells, and a viability of more than $40 \%$.

\section{Data Analysis}

Effect of Different Eosinophil Cut Points

The reproducibility of inflammatory subtype was examined for a series of equally spaced cut points above $1 \%$ eosinophils and the minimum cut point with good reproducibility was used in subsequent analyses. Cut points were incremented by $0.25 \%$ (equivalent to an increase of 1 eosinophil counted in 400 nonsquamous cells from a sputum cytospin) until all patients were classified as having non-eosinophilic asthma. The minimum cut point with good reproducibility was defined as the cut point where further increments provided no or little improvement on the value of the $\kappa$ statistic as calculated using the $\kappa$ statistic derived by Fleiss et al. [11] for multiple raters and 2 categories. Cohen noted that some of the agreement that occurs between raters will have occurred due to chance alone. Therefore, he defined the $\kappa$ statistic as follows: the observed agreement between 2 raters minus the agreement that occurs by chance alone divided by 1 minus the agreement that occurs by chance alone. When there are more than 2 raters the $\kappa$ is calculated as a weighted average of the pairs of raters [11].

Minimum Number of Visits to Confirm Inflammatory

Subtype

Each participant was classified as having eosinophilic or noneosinophilic asthma based upon the most frequently occurring inflammatory subtype over the 5 -visit period using a cut point of $3 \%$ eosinophils. The proportion of participants with an eosinophilic or non-eosinophilic inflammatory subtype when fewer sputum samples were used ( 3 or 1 ) was also compared to the subtype after 5 visits (gold standard) using sensitivity and specificity analysis. That is, we calculated the number of subjects with eosinophilic asthma as determined by the data from 3 visits as a percentage of the total number of subjects classified as having eosinophilic asthma using data from 5 visits (sensitivity), and the number of subjects with non-eosinophilic asthma as determined by the data from 3 visits as a percentage of the number with noneosinophilic asthma from 5 visits (specificity).

\section{Results}

An induced sputum sample was collected on 122 of the 130 clinic visits, which corresponds to a success rate of $94 \%$. Nine (7.4\%) of the 122 sputum samples were inadequate due to low cell viability and were excluded from the analysis. Seventeen (65\%) participants produced an adequate sputum sample at all 5 consecutive monthly clinic visits. The clinical characteristics of the 26 participants are shown in table 1 . 
Table 1. Characteristics of all participants and by inflammatory subtype

\begin{tabular}{|c|c|c|c|c|}
\hline & Total & Non-eosinophilic & Eosinophilic & $\mathrm{p}$ \\
\hline Participants & 26 & 18 & 8 & \\
\hline Age, years & $54 \pm 14$ & $53 \pm 13$ & $55 \pm 17$ & 0.676 \\
\hline Gender, male & 15 & 11 & 4 & 0.457 \\
\hline Atopy, n & $23(88 \%)$ & $16(89 \%)$ & $7(88 \%)$ & 0.686 \\
\hline $\mathrm{FEV}_{1} \%$ predicted & $69 \pm 22$ & $66 \pm 21$ & $76 \pm 24$ & 0.269 \\
\hline $\mathrm{FEV}_{1}: \mathrm{FVC} \%$ & $65 \pm 12$ & $64 \pm 11$ & $69 \pm 13$ & 0.308 \\
\hline Median ICS, $\mu \mathrm{g} /$ day & $2,000(1,000-2,000)$ & $2,000(1,000-2,000) \mathrm{n}=16$ & $1,000(1,000-2,000) \mathrm{n}=5$ & 0.693 \\
\hline
\end{tabular}

Figures in parentheses are interquartile ranges, unless indicated otherwise. Inhaled corticosteroid (ICS) dose is calculated as $1 \mu \mathrm{g}$ of beclomethasone $=1 \mu \mathrm{g}$ budesonide $=0.5 \mu \mathrm{g}$ fluticasone.

The Effect of Different Eosinophil Cut Points

Increasing the cut point from $1 \%$ resulted in a sharp increase in $\kappa$ statistic until approximately $3 \%$ eosinophils (fig. 1). Lower cut points between 1.00 and $1.75 \%$ resulted in a $\kappa$ statistic below 0.4 , which according to the Landi and Koch classification indicated slight or fair agreement. The $\kappa$ statistics for cut points above $2 \%$ were more than 0.4 indicating moderate agreement with a cut point of $3 \%$ eosinophils, producing a $\kappa$ statistic of $0.538(\mathrm{p}<0.0001)$ with little additional benefit of using higher cut points. A cut point of $3 \%$ was chosen for further evaluation.

\section{Minimum Number of Visits to Determine \\ Inflammatory Subtype}

With a cut point of $3 \%$ we then sought to examine the minimum number of visits required to accurately determine the inflammatory subtype. Two (7.7\%) participants did not have an adequate sputum sample for at least 3 visits. Ten (38\%) participants returned the same inflammatory subtype for 5 visits and 10 (38\%) participants who had more than 1 adequate sputum sample changed the subtype during the study (table 2).

When 3 visits (first 3 visits where sputum was obtained) were used to determine the inflammatory subtype the sensitivity was $93 \%$ and the specificity was $100 \%$ when compared to the assignment of inflammatory subtype after 5 visits (gold standard), indicating that assignment of inflammatory subtype after 3 visits is both sensitive and specific. When 1 sputum sample was used to determine the inflammatory subtype there was no change in sensitivity and a small reduction in specificity to $88 \%$ (table 3 ).

Two (9\%) participants would be incorrectly classified if 1 visit were used to determine inflammatory subtype, while 1 (5\%) participant would be over-treated if 3 visits were used.

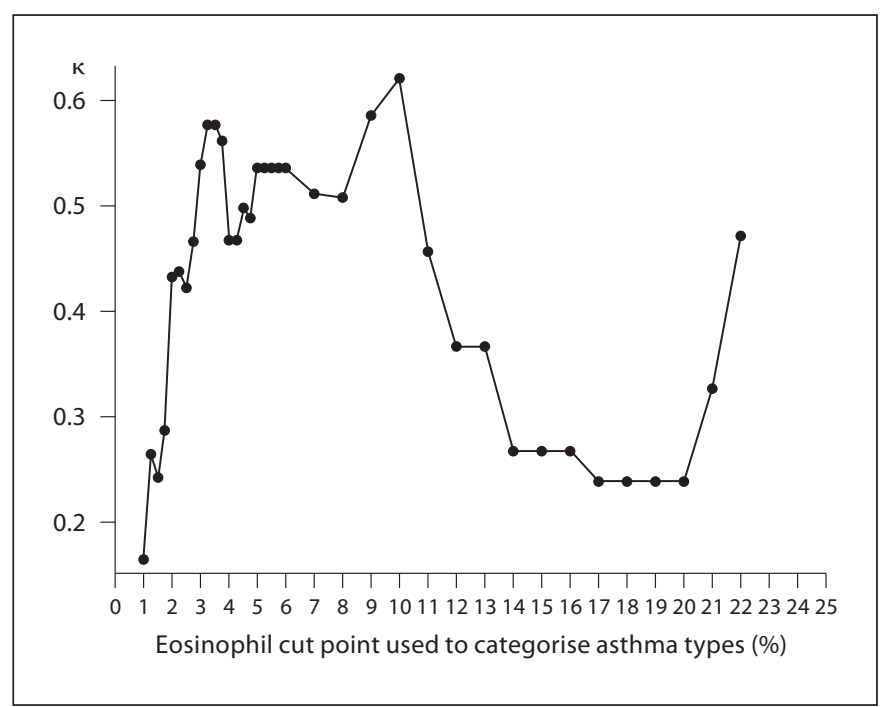

Fig. 1. Agreement (к) in inflammatory subtype using cut points increasing from 1.00 to sputum eosinophils.

\section{Discussion}

This study examined the reproducibility of non-eosinophilic asthma using different eosinophil cut points. There was a high success of sputum induction and 65\% of patients produced an adequate sputum sample each month for 5 months. A cut point of 3\% eosinophils had the optimum performance characteristics for assessment of inflammatory subtype. Also, a cut point of 3\% was more reproducible and a single sputum sample was sufficient to assign inflammatory subtype. This has important clinical implications for the use of induced sputum in both clinical and research practice. 
Table 2. Sputum eosinophil proportion (inflammatory subtype in parentheses) for each participant at each visit

\begin{tabular}{|c|c|c|c|c|c|}
\hline ID & Visit 1 & Visit 2 & Visit 3 & Visit 4 & Visit 5 \\
\hline N003 & $13.00(\mathrm{EA})$ & $11.25(\mathrm{EA})$ & $21.00(\mathrm{EA})$ & $10.00(\mathrm{EA})$ & 16.00 (EA) \\
\hline N004 & 1.00 (NEA) & 0.50 (NEA) & 0.25 (NEA) & 1.00 (NEA) & 0.00 (NEA) \\
\hline N005 & 1.50 (NEA) & 7.50 (EA) & 1.00 (NEA) & 0.00 (NEA) & 0.00 (NEA) \\
\hline N010 & * & 3.50 (EA) & 0.00 (NEA) & 0.75 (NEA) & 0.75 (NEA) \\
\hline N019 & 0.00 (NEA) & 1.25 (NEA) & 1.75 (NEA) & * & 1.25 (NEA) \\
\hline N023 & * & * & * & * & 26.50 (EA) \\
\hline N024 & * & 0.25 (NEA) & 0.75 (NEA) & * & 1.80 (NEA) \\
\hline N029 & 3.60 (EA) & 7.25 (EA) & & $11.75(\mathrm{EA})$ & \\
\hline N032 & * & 0.00 (NEA) & 0.50 (NEA) & $4.25(\mathrm{EA})$ & 0.75 (NEA) \\
\hline N033 & 0.00 (NEA) & 0.00 (NEA) & 0.25 (NEA) & 3.00 (EA) & 1.00 (NEA) \\
\hline N035 & 1.25 (NEA) & $2.00(\mathrm{NEA})$ & 1.25 (NEA) & 2.50 (NEA) & \\
\hline N037 & 0.00 (NEA) & 0.50 (NEA) & 1.75 (NEA) & 1.50 (NEA) & 4.75 (EA) \\
\hline N047 & 0.00 (NEA) & 0.25 (NEA) & 0.00 (NEA) & 0.00 (NEA) & 0.50 (NEA) \\
\hline N050 & 0.00 (NEA) & 0.00 (NEA) & 0.25 (NEA) & 0.00 (NEA) & 1.75 (NEA) \\
\hline N062 & 0.40 (NEA) & 1.50 (NEA) & 0.71 (NEA) & 0.50 (NEA) & 0.50 (NEA) \\
\hline N063 & 3.75 (EA) & $21.75(\mathrm{EA})$ & $37.25(\mathrm{EA})$ & 0.50 (NEA) & $7.25(\mathrm{EA})$ \\
\hline N067 & 3.75 (EA) & $8.50(\mathrm{EA})$ & 20.25 (EA) & $6.50(\mathrm{EA})$ & 3.75 (EA) \\
\hline N073 & 9.25 (EA) & 2.40 (NEA) & & & \\
\hline N074 & 2.25 (NEA) & 0.80 (NEA) & & 0.00 (NEA) & \\
\hline N080 & 0.75 (NEA) & 4.25 (EA) & 0.50 (NEA) & 1.40 (NEA) & 0.25 (NEA) \\
\hline N081 & 0.50 (NEA) & 0.25 (NEA) & 2.75 (NEA) & 2.50 (NEA) & 0.75 (NEA) \\
\hline N084 & 3.75 (EA) & 1.75 (NEA) & 3.75 (EA) & 0.50 (NEA) & 2.25 (NEA) \\
\hline N085 & 2.75 (NEA) & 0.00 (NEA) & 1.00 (NEA) & 0.50 (NEA) & 2.25 (NEA) \\
\hline N087 & 1.25 (NEA) & 1.00 (NEA) & 0.25 (NEA) & 0.00 (NEA) & 1.75 (NEA) \\
\hline N095 & 8.75 (EA) & $4.50(\mathrm{EA})$ & 0.25 (NEA) & 4.50 (EA) & 4.25 (EA) \\
\hline N099 & 1.75 (NEA) & 0.75 (NEA) & 0.25 (NEA) & 0.00 (NEA) & 0.25 (NEA) \\
\hline
\end{tabular}

EA = Eosinophilic asthma, sputum eosinophils $\geq 3 \%$; NEA = non-eosinophilic asthma, sputum eosinophils $<3 \%$. No result indicates the participant did not produce a sputum sample and asterisks indicate the sputum sample had poor viability (viability $<40$ ) and therefore the results were not included in the analysis.

Table 3. Two by two table showing the number of participants classified as eosinophilic asthma and non-eosinophilic asthma

\begin{tabular}{ll}
5 visits \\
\hline EA NEA \\
\hline
\end{tabular}

After 3 visits compared to 5 visits

$\begin{array}{lllrr}3 \text { visits } & \text { EA } & 4 & 1 & 5 \\ & \text { NEA } & 0 & 14 & 14 \\ & & 4 & 15 & 19\end{array}$

After 1 visit compared to 5 visits

$\begin{array}{llrrr}1 \text { visit } & \text { EA } & 5 & 2 & 7 \\ & \text { NEA } & 0 & 15 & 15 \\ & & 5 & 17 & 22\end{array}$

$\mathrm{EA}=$ Eosinophilic asthma; NEA = non-eosinophilic asthma.
A cut point of $3 \%$ eosinophils was used to guide treatment decisions in 2 randomised controlled trials of patients with airway disease. Patients with asthma experienced significantly fewer severe exacerbations and had a $63 \%$ reduction in sputum eosinophil count when corticosteroid treatment was increased when sputum eosinophils were more than 3\% [6]. A similar treatment protocol was used in patients with COPD resulting in a reduction in severe exacerbations requiring hospitalisation [12]. In both studies, treatment was not altered if the sputum eosinophil proportion was between 1 and 3\%, and treatment was reduced where the patients' sputum had less than $1 \%$ eosinophils. A study investigating the ability of sputum eosinophils to predict a clinical response to corticosteroids has demonstrated that a baseline eosinophil proportion of more than $3 \%$ predicted the best $\mathrm{FEV}_{1}$ responses to corticosteroid treatment [13]. In this study, there was 
poor reproducibility in the assignment of inflammatory subtype when cut points were between 1 and $1.75 \%$. Moderate agreement was achieved in all cut points above $2 \%$ and the highest agreement was observed with a cut point of $3 \%$.

This research was conducted over 5 months, which allowed an investigation of the reproducibility of the inflammatory subtype; however, this is not a clinically practical timeframe and 3 or fewer visits are more likely to be used to determine treatment requirements for a patient. Compared to this gold standard of inflammatory subtype determined after 5 sputum samples, using 3 consecutive sputum samples was both specific and sensitive. However, a single sputum sample resulted in the correct assignment of all patients with eosinophilic asthma and an incorrect assignment of 2 patients with non-eosinophilic asthma. In clinical practice this could result in the patient being prescribed corticosteroids.

The utility of managing asthma using inflammatory subtype has recently been established in 2 randomised controlled trials. Both studies show how management strategies which minimise eosinophilia reduce severe exacerbations $[6,7]$. Because inhaled corticosteroids are ineffective in the treatment of non-eosinophilic asthma [14], current management strategies could be providing unnecessary, ineffective treatment. In fact, in the study conducted by Green et al. [6], a subgroup of patients with non-eosinophilic asthma managed using both clinical and inflammatory control measures reduced their inhaled corticosteroid dose by more than $900 \mu \mathrm{g}$ per day.

This study is limited in its small sample size of 26 participants. Larger prospective studies are needed to examine the reproducibility of inflammatory subtypes more closely. It would be especially important to understand the variability in inflammatory subtype over a longer period where exacerbations are experienced to determine if the exacerbation may result in an alteration of the inflammatory subtype.

In conclusion, the choice of a cut point of $3 \%$ eosinophils to distinguish eosinophilic from non-eosinophilic asthma has many advantages. Firstly, it is clearly outside the normal range of healthy patients without airway disease. Secondly, it is associated with a positive treatment effect, as patients with eosinophil proportions higher than $3 \%$ are responsive to inhaled corticosteroids. Thirdly, this study shows that a cut point of $3 \%$ is stable and reproducible over a period of 5 months and a single visit is sufficient to detect the presence of eosinophilic inflammation. We recommend that an induced sputum proportion of $3 \%$ be used to identify patients with eosinophilic airway inflammation.

\section{Acknowledgment}

This study was supported by a National Health and Medical Research Council project grant.

\section{References}

1 Pavord ID, Brightling CE, Woltmann G, Wardlaw AJ: Non-eosinophilic corticosteroid unresponsive asthma. Lancet 1999;353: 2213-2214.

-2 Turner MO, Hussack P, Sears MR, Dolovich J, Hargreave FE: Exacerbations of asthma without sputum eosinophilia. Thorax 1995; 50:1057-1061.

3 Lex C, Payne DN, Zacharasiewicz A, Li AM, Wilson NM, Hansel TT, Bush A: Sputum induction in children with difficult asthma: safety, feasibility, and inflammatory cell pattern. Pediatr Pulmonol 2005;39:318-324.

-4 Simpson JL, Scott R, Boyle M, Gibson P: Inflammatory subtypes in asthma: assessment and identification using induced sputum. Respirology 2006;11:54-61.

-5 Wenzel SE, Schwartz LB, Langmack EL, Halliday JL, Trudeau JB, Gibbs RL, Chu HW: Evidence that severe asthma can be divided pathologically into two inflammatory subtypes with distinct physiologic and clinical characteristics. Am J Respir Crit Care Med 1999;160:1001-1008.
6 Green RH, Brightling CE, McKenna S, Hargadon B, Parker D, Bradding P, Wardlaw AJ, Pavord ID: Asthma exacerbations and sputum eosinophil counts: a randomised controlled trial. Lancet 2002;30:1715-1721.

7 Jayaram L, Pizzichini MM, Cook RJ, Boulet LP, Lemiere C, Pizzichini E, Cartier A, Hussack P, Goldsmith CH, Laviolette M, Parameswaran K, Hargreave FE: Determining asthma treatment by monitoring sputum cell counts: effect on exacerbations. Eur Resp J 2006;27:483-494.

-8 Simpson JL, Powell H, Boyle MJ, Scott RJ, PG G: Clarithromycin targets neutrophilic inflammation in refractory asthma. Am J Resp Crit Care Med 2007;177:148-155.

9 Global Initiative for Asthma (GINA): Global Strategy for Asthma Management and Prevention Report. Bethesda, National Heart, Lung, and Blood Institute, National Institutes of Health, 2006.

10 Gibson PG, Wlodarczyk JW, Hensley MJ, Gleeson M, Henry RL, Cripps AR, Clancy RL: Epidemiological association of airway inflammation with asthma symptoms and airway hyperresponsiveness in childhood. Am J Resp Crit Care Med 1998;158:36-41.

11 Fleiss JL, Nee CM, Landis JR: Large sample variance of $\kappa$ in the case of different sets of raters. Psychol Bull 1979;86:974-977.

12 Siva R, Green RH, Brightling CE, Shelley M, Hardagon D, McKenna S, Monteiro W, Berry M, Parker D, Wardlaw AJ, Pavord ID: Eosinophilic airway inflammation and exacerbations of COPD: a randomised controlled trial. Eur Respir J 2007;29:906-913.

13 Meijer RJ, Postma DS, Kauffman HF, Arends LR, Koeter GH, Kerstjens HAM: Accuracy of eosinophils and eosinophilic cationic protein to predict steroid improvement in asthma. Clin Exp Allergy 2002;32:10961103.

14 Berry MA, Morgan A, Shaw DE, Parker D, Green RH, Brightling CE, Bradding P, Wardlaw AJ, Pavord ID: Pathological features and inhaled corticosteroid response of eosinophilic and non-eosinophilic asthma. Thorax 2007;62:1043-1049. 